TITLE:

\title{
Temperature dependence of terahertz emission by an asymmetric intrinsic Josephson junction device
}

$\operatorname{AUTHOR}(\mathrm{S})$ :

Kakeya, Itsuhiro; Hirayama, Nobuo; Omukai, Yuta; Suzuki, Minoru

\section{CITATION:}

Kakeya, Itsuhiro ... [et al]. Temperature dependence of terahertz emission by an asymmetric intrinsic Josephson junction device. Journal of Applied Physics 2015, 117(4): 043914.

ISSUE DATE:

2015-01-30

URL:

http://hdl.handle.net/2433/216403

\section{RIGHT:}

(c) 2015 AIP Publishing LLC. This article may be downloaded for personal use only. Any other use requires prior permission of the author and AIP Publishing. The following article may be found at

http://scitation.aip.org/content/aip/journal/jap/117/4/10.1063/1.4906849; The full-text file will be made open to the public on 30 January 2016 in accordance with publisher's 'Terms and Conditions for Self-Archiving'. 


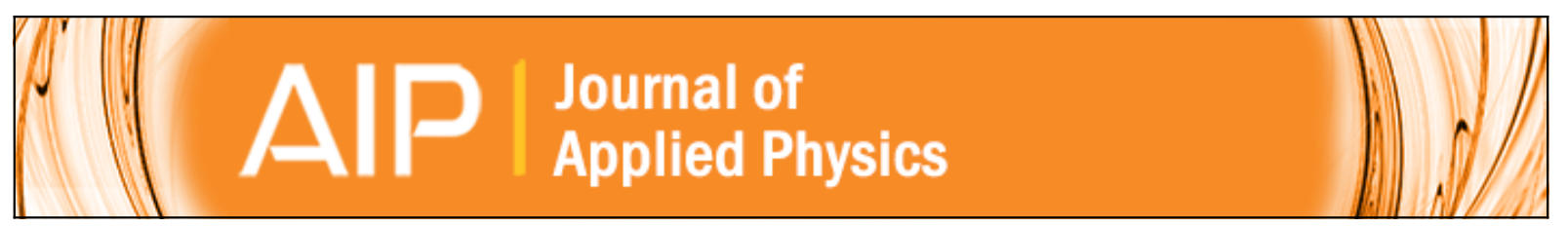

Temperature dependence of terahertz emission by an asymmetric intrinsic Josephson junction device

Itsuhiro Kakeya, Nobuo Hirayama, Yuta Omukai, and Minoru Suzuki

Citation: Journal of Applied Physics 117, 043914 (2015); doi: 10.1063/1.4906849

View online: http://dx.doi.org/10.1063/1.4906849

View Table of Contents: http://scitation.aip.org/content/aip/journal/jap/117/4?ver=pdfcov

Published by the AIP Publishing

\section{Articles you may be interested in}

Intense terahertz emission from intrinsic Josephson junctions by external heat control

Appl. Phys. Lett. 104, 112601 (2014); 10.1063/1.4868219

Terahertz-wave radiation emitted by intrinsic Josephson junctions

J. Appl. Phys. 107, 103920 (2010); 10.1063/1.3393037

Josephson vortices as flexible waveguides for terahertz waves

J. Appl. Phys. 104, 064507 (2008); 10.1063/1.2979714

Vortex-flow electromagnetic emission in stacked intrinsic Josephson junctions

Appl. Phys. Lett. 88, 142501 (2006); 10.1063/1.2191415

Josephson fluxon flow and phase diffusion in thin-film intrinsic Josephson junctions

J. Appl. Phys. 95, 4941 (2004); 10.1063/1.1689754

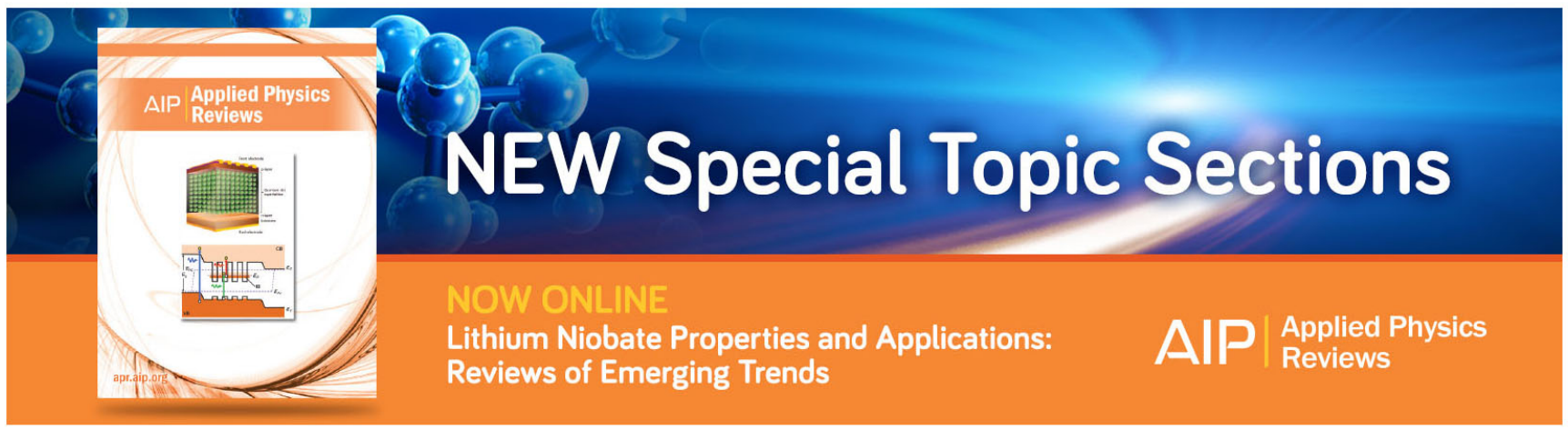




\title{
Temperature dependence of terahertz emission by an asymmetric intrinsic Josephson junction device
}

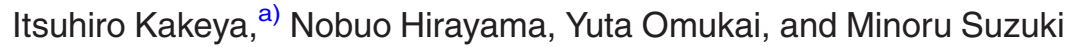 \\ Department of Electronic Science and Engineering, Kyoto University, Nishikyo-ku, Kyoto 615-8510, Japan
}

(Received 5 November 2014; accepted 16 January 2015; published online 30 January 2015)

\begin{abstract}
This study investigates the effect of temperature on the emission frequency of an intrinsic Josephson junction terahertz (THz) electromagnetic wave source, which can be used for high-speed communications by $\mathrm{THz}$ carrier wave. The characteristic emission features of two device types (asymmetric and symmetric) and two bias regimes (low and high) were determined. The biasdependent emission frequency was temperature dependent in the asymmetric device, most likely reflecting the temperature-dependent London penetration depth. The bias tunability of the emission frequency can be explained by device self-heating, which significantly and inhomogeneously raises the temperatures of the device from its bath temperature. These findings are consistent with previous studies of temperature distribution in these devices. (C) 2015 AIP Publishing LLC.
\end{abstract}

[http://dx.doi.org/10.1063/1.4906849]

\section{INTRODUCTION}

Stacks of intrinsic Josephson junctions (IJJs) constructed from the high- $T_{c}$ superconductor $\mathrm{Bi}_{2} \mathrm{Sr}_{2} \mathrm{CaCu}_{2} \mathrm{O}_{8+\delta}(\mathrm{Bi} 2212)$ emit at terahertz (THz) frequencies. Such IJJ emission has rapidly attracted theoretical and experimental interest. ${ }^{1-8}$ The emitted terahertz waves are considered to be monochromatic and coherent because it is attributed to synchronization of stacked Josephson junctions naturally formed inside Bi2212 single crystals. Thus, these IJJs can be used for generating $\mathrm{THz}$ waves to realize ultrafast and huge-capacity wireless data transfers. The emission frequencies of these structures (ranging from 0.3 to $0.9 \mathrm{THz}$ ) are roughly determined by the geometry of the stacks. Reportedly, the emission power of a single stack of coherently oscillating IJJs and synchronized three stacks can reach $30 \mu \mathrm{W}$ (Refs. 9 and 10 ) and $0.6 \mathrm{~mW},{ }^{11}$ respectively. The underlying physics behind these phenomena is synchronization of hundreds of IJJs consisting of a stack and synchronization of stacks connected by a base crystal.

To understand the synchronization mechanism, we must elucidate the temperature distribution in the stack. Since the self-generated heat in the voltage state is retained by the low thermal conductivity of the superconducting crystal, localized temperature rise in the stack is inevitable. ${ }^{12}$ Local temperature rise above the critical temperature $T_{c}$ (hotspots) of the emitting devices has been first identified by lowtemperature scanning laser microscopy (LTSLM). ${ }^{5}$ Although the LTSLM signals reflect changes in the various physical parameters of the stacks, these results predict that coherent oscillation along the $c$-axis arises from spatial inhomogeneity of the junction along the $a b$-plane. Meanwhile, we previously altered the thickness of the electrodes atop the stacks, and found that emission is largely governed by thermal inhomogeneity of the emitting stack itself, which is caused by the weak heat link between the stack and thermal bath. ${ }^{13}$ More recently, we have reported that the hotspots are detrimental for emission intensities. ${ }^{14}$

a)kakeya@kuee.kyoto-u.ac.jp
The emission frequency $f_{e}$ is tuned by changing either the bias current or bath temperature $T_{b}$. Benseman et al. investigated the temperature dependence of the emission frequency in asymmetric device configuration, namely, a superconducting substrate overlain with a trapezoidal stack. They attributed the frequency-temperature relation to the temperature dependence of the London penetration depth along the $a b$-plane. ${ }^{15}$ At a certain temperature, an IJJ included in the stack might entrain IJJs of different resonance frequencies (widths) due to the trapezoidal stack shape, establishing a tuning bandwidth. ${ }^{16}$ The amplitude of the Josephson oscillation has a node at the superconducting substrate because the substrate and stacked IJJs are in the superconducting (zero-voltage) and voltage state, respectively.

Two emission regimes have been recognized: a low-bias regime well below the critical current in the voltage state, and a high-bias regime, in which the current exceeds the critical current. The high-bias regime admits a rich variety of phenomena, such as wide-range frequency tuning, hotspot formation, and a sharp spectrum linewidth, whereas less complicated behavior is observed in the low-bias regime. ${ }^{17-19}$ Although the high-bias emission presumably linked to temperature inhomogeneity, no systematic difference between the frequency tuning of high-bias and low-bias emissions has been reported to date.

In this letter, we discuss how the emission frequency $f_{e}$ is affected by temperature in three devices. Low-bias emission from the mesa device is explained by the $T_{b}$ dependence of the London penetration depth, whereas the high-bias emission is attributed to significant temperature rise from $T_{b}$ and temperature inhomogeneity in the device. Different from the asymmetric mesa device, low-bias emission from the symmetric stand-alone stack is independent of $T_{b}$ because no nodal Josephson oscillation is established along the $c$-axis.

\section{EXPERIMENTS}

We prepared three devices from $\mathrm{Bi}_{2} \mathrm{Sr}_{2} \mathrm{CaCu}_{2} \mathrm{O}_{8+\delta}$ single crystals grown by the traveling solvent floating zone method. Device A and C are ordinary mesa devices comprising IJJ stacks formed on bulk single crystals by 


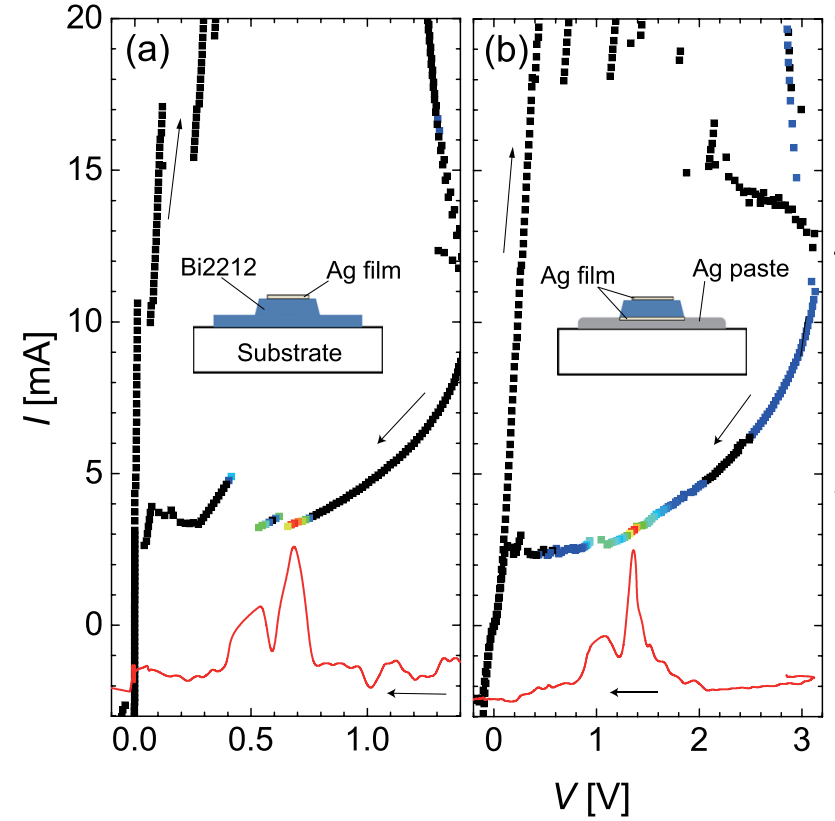

photolithography and the Ar milling technique as described in the previous works. Device B is a stand-alone stack. ${ }^{18}$ An IJJ stack was removed from a bulk crystal and glued onto a sapphire substrate with silver epoxy. The stack was sandwiched between a pair of silver electrodes formed by a vacuum evaporation technique. Schematics of the three devices are depicted in the insets of Fig. 1. The stacks were approximately $400 \mu \mathrm{m}$ long, but their widths $w$ and thicknesses $t$ ranged from 60 to $90 \mu \mathrm{m}$ and 1.1 to $1.8 \mu \mathrm{m}$, respectively. The geometries of the devices are summarized in Table I.

To precisely determine the temperature dependence of $f_{e}$, we measured emission spectra of the devices by a laboratory-designed high-resolution FT-IR spectrometer ${ }^{20}$ after checking $\mathrm{THz}$ wave emission using a simple detection system. ${ }^{13}$ The frequency resolution of the spectrometer is less than $1 \mathrm{GHz}$, an order of magnitude higher than that of an ordinary commercial FT-IR spectrometer, enabling precise determination of the temperature effect on $f_{e}$. The bath temperature $T_{b}$ was measured at the copper sample stage, to which the device was glued with silver paste. The devices on their copper stages were placed in a vacuum and the emitted $\mathrm{THz}$ waves passed through a white polyethylene window.

\section{RESULTS AND DISCUSSION}

\section{A. Device A: Low bias regime}

$\mathrm{THz}$ frequencies were emitted from Device A within a limited bias region in the low-bias regime, as shown in

TABLE I. List of samples used in this study. $w_{t}$ and $w_{b}$ represent the top and bottom widths of the stack, respectively. For the analysis, the averaged width $w=\left(w_{t}+w_{b}\right) / 2$ is used in the text. The stack thickness $t$ and the critical temperature $T_{c}$ of the device are shown.

\begin{tabular}{cccccc}
\hline \hline & Type & $w_{t}(\mu \mathrm{m})$ & $w_{b}(\mu \mathrm{m})$ & $t(\mu \mathrm{m})$ & $T_{c}(\mathrm{~K})$ \\
\hline A & Mesa & 82.1 & 92.3 & 1.19 & 72 \\
B & Stand-alone & 64.5 & 75.5 & 1.80 & 70 \\
$\mathrm{C}$ & Mesa & 65.5 & 74.3 & 1.11 & 91.5 \\
\hline \hline
\end{tabular}

Fig. 1(a). The emission was observed between 15 and $45 \mathrm{~K}$ just above the discontinuity in the $I-V$ characteristics (retrap). The emission range is $0.5<V<0.8 \mathrm{~V}$ and $3<I<4 \mathrm{~mA}$, and the detected intensity roughly decreases with increasing $T_{b}$. The emission spectra at various $T_{b} \mathrm{~s}$ are shown in Fig. 2(a). At $25 \mathrm{~K}$, the peak frequency and spectrum width are 450 and $2.2 \mathrm{GHz}$, respectively. Through the AC Josephson relation $2 e v=\hbar \omega$, the frequency $\omega / 2 \pi$ corresponds to a junction voltage $v=0.92 \mathrm{mV} / \mathrm{IJJ}$. Thus, the total voltage across the device is $N v=0.74 \mathrm{~V}$, where $N$ is the number of IJJs $(N \simeq 800)$, which approximately matches the bias voltage at the peak intensity $V_{e}=0.76 \mathrm{~V}$. As $T_{b}$ increases, $f_{e}$ decreases, maintaining the AC Josephson relation within a few percent. The spectrum width is independent of $T_{b}$ and is narrower than that obtained by heterodyne

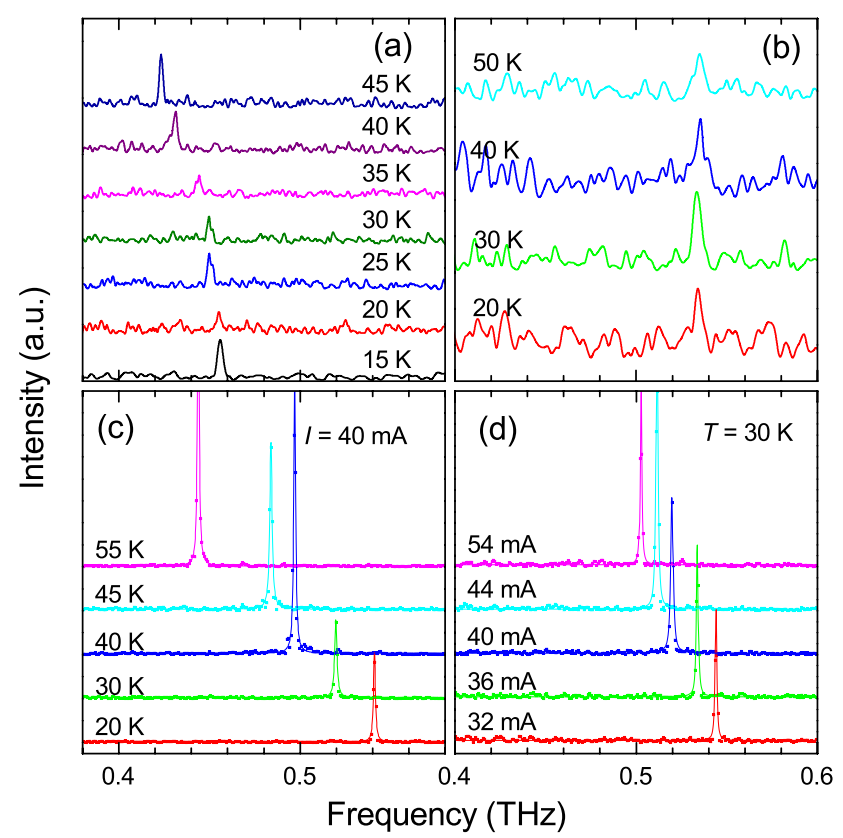

FIG. 2. Emission spectra at various $T_{b} \mathrm{~s}$ in device A (a), B (b), and C (c). Panel (d) shows the bias dependence of the spectra in device $\mathrm{C}$. 
mixing, in which poor coherence of the low-bias emission has been claimed. ${ }^{19}$

The temperature dependence of $f_{e}$ is plotted in Fig. 3(a). Temperature-frequency relations of the standing waves in a resonator are given by temperature dependence of the propagation velocity of electromagnetic waves inside the resonator (the so-called Swihart velocity for a single Josephson junction). Josephson plasma waves with both $k_{x}$ (parallel to the $a b$-plane) and $k_{z}$ (parallel to the $c$-axis) may be excited in an IJJ stack; thus, the propagation velocity is estimated as $\tilde{c}^{2}=c_{0}^{2}\left[\epsilon_{c} 2\left\{1-\cos \left(k_{z} s\right)\right\} \lambda_{a b} / s^{2}+1\right]^{-1}$, where $k_{z}, \lambda_{a b}$, and $s$ are the wavenumber of the standing wave along the $c$-axis, the London penetration depth along the $a b$-plane, and the thickness of the superconducting layers, respectively. ${ }^{21}$ Benseman et al. suggested that if the boundary conditions of the stack are asymmetric with respect to the $c$-axis, an asymmetric standing wave is established along the $c$-axis with $k_{z}=m \pi / 2 t(m=1,3, \ldots)$, where $t$ is the thickness of the stack.

Since the length of the stack is sufficiently longer than the width, standing wave with $k_{x}$ along the width is dominant. Furthermore, $2 \pi / k_{z}=2 t \sim 10^{3} \mathrm{~s}$ and $f_{e} \gg \omega_{p} / 2 \pi$ hold. Finally, the frequency of the two-dimensional standing wave is given by

$$
f=\frac{c_{0}}{2 w \sqrt{\epsilon_{c}\left\{1+\left(m \pi \lambda_{a b}(T) / 2 t\right)^{2}\right\}}} .
$$

The frequency $f$ depends on temperature because $\lambda_{a b}(T)$ is a temperature-dependent parameter, as measured by several authors. $^{22-24}$ The solid curve in Fig. 3(a) plots Eq. (1) as a function of temperature, using the data of Anukool et al. data with the amount of doping $p=0.124$ (determined by the $T_{c}$ of the Device A). ${ }^{22}$ Setting the refractive index $n=\sqrt{\epsilon_{c}}=3.35, m=1$, and $w=87 \mu \mathrm{m}$, an excellent agreement is obtained between Eq. (1) and the experimental data.

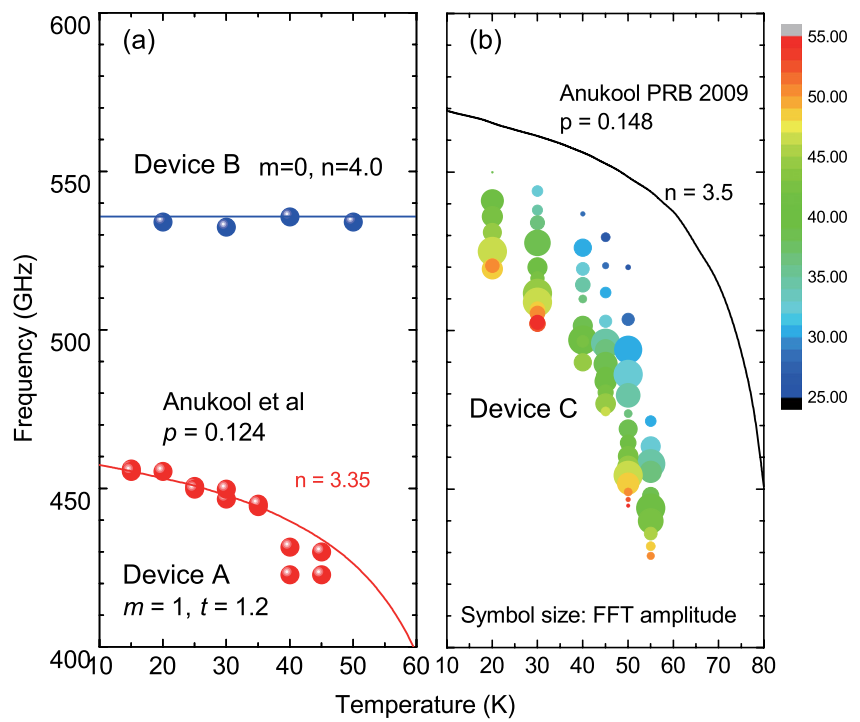

FIG. 3. Temperature dependence of the emission frequency from devices (a) A, B, and (b) C. Solid lines are values of Eq. (1) with parameters given in the figures. Data of $\lambda_{a b}(T)$ are obtained from Ref. 22 with close $T_{c}$. Symbol color in (b) represents bias current according to the color bar at right and symbol size means FFT amplitude of the spectra.

\section{B. Device B: Stand-alone IJJ stack}

The $I-V$ characteristics and emission intensities of Device B are plotted in Fig. 1(b). The intensity sharply peaks at $1.38 \mathrm{~V}$ and a weaker peak appears at $1.10 \mathrm{~V}$. Device B emitted $\mathrm{THz}$ radiation in the low-bias regime. Figure 2(b) shows the FT-IR spectra taken at optimum bias points at various temperatures. Note that $f_{e}(\simeq 540 \mathrm{GHz})$ is independent of temperature between 10 and $50 \mathrm{~K}$ in a sharp contrast to mesa-type devices, whose emission frequencies decrease with increasing $T_{b}$.

We interpret the insensitivity of $f_{e}$ to the temperature in Device B as follows. The highly conducting silver electrodes are affixed to the top and bottom of the stand-alone stack; moreover the whole stack possibly becomes uniformly resistive because of the absence of superconducting substrate. The symmetric stack configuration admits excitation of the $m=0$ uniform mode rendering Eq. (1) independent of $\lambda_{a b}(T)$. Through the AC Josephson relation, $f \simeq 540 \mathrm{GHz}$ corresponds to $1.11 \mathrm{mV}$ per IJJ, resulting in $1.35 \mathrm{~V}$ across the whole stack of $N=1200$. This voltage is quite close to prominent peak bias voltage $(1.38 \mathrm{~V})$, supporting that $m=0$ standing waves are excited along the $c$-axis.

The refractive index $n=c_{0} / 2 w f_{e}$ was considerably higher in Device B than in the other two devices, presumably because the effective width of the stack was considerably shorter than $w$ in this device. The edges and surfaces of stand-alone stack are more deteriorated by organic solvents and water than those of typical mesa devices, because of the complicated fabrication process and the large surface area. Similar results for stand-alone stacks and focused-ion-beammilled mesas have been reported in previous studies. ${ }^{18,25}$

\section{Device C: High-bias regime}

Device $\mathrm{C}$ demonstrates high-bias emission. The highbias regime covers the region $30<I<60 \mathrm{~mA}$ and $0.7<V<0.9 \mathrm{~V}$, as shown in Fig. 1(c). Figures 2(c) and 2(d) present the spectra of the high-bias emissions at various temperatures with a constant bias current of $40 \mathrm{~mA}$, and at various bias currents with $T_{b}=30 \mathrm{~K}$, respectively. As the temperature increases from 20 to $60 \mathrm{~K}, f_{e}$ decreases from 0.54 to $0.43 \mathrm{THz}$. Increasing the bias current similarly decreases the $f_{e}$. From this result, we attribute the decrease in $f_{e}$ to the increase in effective temperature of the stack as $\tilde{c}$ decreases. The emission frequencies at different $T_{b}$ and bias conditions are plotted in Fig. 3(b). Obviously, $f_{e}$ decreases as either $T_{b}$ or bias increases; however, these experimental results are considerably lower than the predictions of Eq. (1) with $\lambda_{a b}(T)$ determined by its $p$ as well as in Device A. The theoretical curve may overlap with the experimental data shifted toward higher temperatures along the temperature axis. It is interpreted that in the high-bias regime, the effective temperature giving rise to $\tilde{c}$ is significantly higher than $T_{b}$, in sharp contrast to the results of Device A.

These results suggest a highly inhomogeneous temperature distribution in the high-bias regime. This is consistent with previous imaging measurements $5,11,14$ and numerical calculations. $^{12,26}$ Figure 4 (a) plots $f_{e}$ (solid symbols) as a function of the $c$-axis biased resistance $\bar{R}_{c}=V / I$, corrected 
by subtracting the contact resistance (included in Fig. 1(c)). Assuming that the $\bar{R}_{c}$ corresponds to the quasiparticle resistance at the effective stack temperature $T>T_{b}$, a homogeneous temperature distribution would yield single values of Eq. (1) and $\bar{R}_{c}(T)$; accordingly, these plots should collapse onto a single line. Instead, the $R_{c}$ reduces as $T_{b}$ is lowered. This deviation can be explained by considering an inhomogeneous temperature distribution and a temperature dependence of both $f(T)$ and $\tilde{c}(T)$ as described in the following paragraph.

As a toy model of an IJJ stack with strong temperature inhomogeneity, we consider two linked IJJ stacks 1 and 2 with the equal volume and uniform temperature $T_{1}$ and $T_{2}$ electrically connected in parallel as shown in Fig. 4(b). This models a device with a hotspot (lower resistance) region shunting a lower temperature (higher resistance) region. The excited electromagnetic wave propagates inside the media with refractive indices $n_{1}$ and $n_{2}$ connected in series without reflection at their interface. $T_{1}\left(T_{2}\right)$ is $\delta T$ higher (lower) than the average temperature $T_{0}$, i.e., $T_{1}=T_{0}+\delta T, T_{2}=T_{0}-\delta T$. The measured resistance $\bar{R}_{c}\left(T_{b}\right)$ is considered as the parallel connection of $R_{c}\left(T_{1}\right)=R_{c}\left(T_{0}\right)-\delta R_{c}$ and $R_{c}\left(T_{2}\right)$ $=R_{c}\left(T_{0}\right)+\delta R_{c}$, where $R_{c}(T)$ represents the intrinsic $c$-axis resistance of the stack at temperature $T$. Consequently, we obtain $\bar{R}_{c}\left(T_{b}\right)=R_{c}\left(T_{0}\right)\left[1-\left(\delta R_{c} / R_{c}\left(T_{0}\right)\right)^{2}\right]$, which shows that decrease in $\bar{R}_{c}\left(T_{b}\right)$ from $R_{c}\left(T_{0}\right)$ is more significant for larger $\delta T$. This situation is more pronounced at lower temperature because the thermal conductivity is smaller. On the other hand, the measured emission frequency $f_{e}$ is

(a)

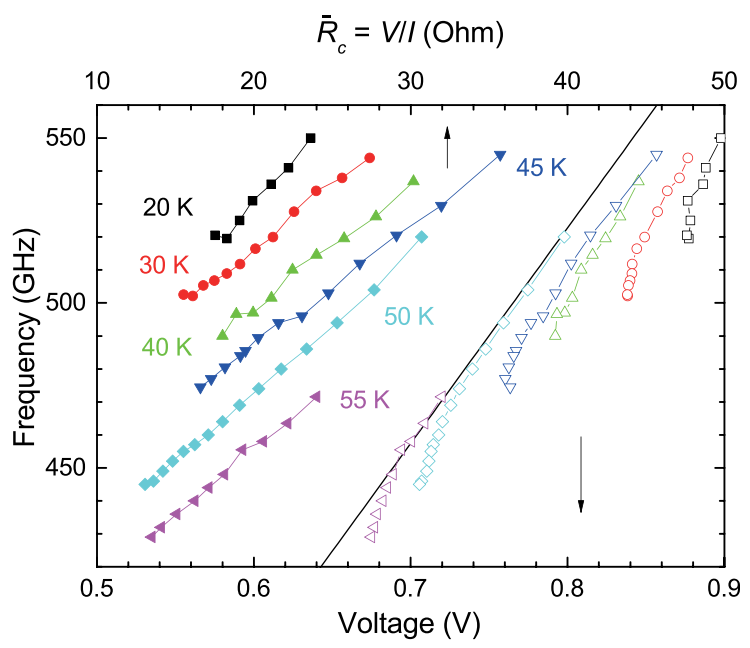

(b)

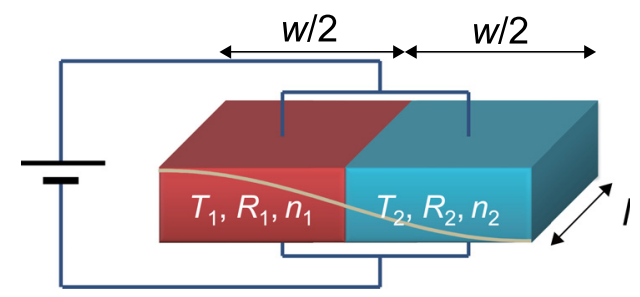

FIG. 4. (a) Plots of emission frequency $f_{e}$ vs averaged resistance $\bar{R}_{c}$ (filled symbols; top axis) and bias voltage $V$ (open symbols; bottom axis) of Device C. The same colors represent data at the same temperature. The solid line is the ac Josephson relation. (b) Toy model of a mesa with an inhomogeneous temperature distribution. A gray solid curve represents the excited electromagnetic wave inside the stack. determined by the distribution in the effective refractive index given by $\tilde{n}(T)=\sqrt{\epsilon_{c}\left\{1+\left(\pi \lambda_{a b}(T) / 2 t\right)^{2}\right\}}$. Assuming $\tilde{n}\left(T_{1}\right)=\tilde{n}\left(T_{0}\right)+\delta \tilde{n}$ and $\tilde{n}\left(T_{2}\right)=\tilde{n}\left(T_{0}\right)-\delta \tilde{n}$, measured refractive index $\bar{n}\left(T_{b}\right)=\left\{\tilde{n}\left(T_{1}\right)+\tilde{n}\left(T_{2}\right)\right\} / 2$. Therefore, we can simply state $\bar{n}\left(T_{b}\right)=\tilde{n}\left(T_{0}\right)$, which corresponds to $f_{e}\left(T_{b}\right)=f\left(T_{0}\right)$ given by Eq. (1); that is, the measured emission frequency $f_{e}$ is directly determined by the average temperature $T_{0}$. From this simplified consideration and comparison with the homogeneous case, we infer that temperature inhomogeneity is responsible for decreases in $\bar{R}_{c}\left(T_{b}\right)$ with respect to $f_{e}\left(T_{b}\right)$. Since the temperature distribution in Bi2212 IJJ stacks emitting at terahertz frequencies becomes more homogeneous at higher temperatures, ${ }^{27}$ the quantity $\bar{R}_{c}\left(T_{b}\right)-$ $R_{c}\left(T_{0}\right)$ due to the temperature inhomogeneity becomes more remarkable at lower $T_{b}$, where $\delta R_{c}$ is larger even for a certain $\delta T$. This conclusion is consistent with the plots in Fig. 4(a), which shift toward lower $\bar{R}_{c}$ (left) as $\delta R_{c}$ increases at lower $T_{b}$. A comparison between $\bar{R}_{c}$ and $R_{c}-T$ data allows to estimate an average temperature $T_{0}$ of the stack. As a result, $T_{0} \mathrm{~s}$ are roughly estimated as 100 and $60 \mathrm{~K}$ for $\bar{R}_{c}=15$ and $30 \Omega$, respectively. The mean-field critical temperature of the device being $91.5 \mathrm{~K}$ also supports the strong temperature inhomogeneity of the device.

The present interpretation does not include the stacking effect of IJJs. Benseman et al., ${ }^{28}$ have discussed the excess $c$ axis voltage with respect to the emission frequency due to temperature inhomogeneity along the $c$-axis. Synchronized IJJs closer to the mesa surface (electrode) arise equal voltage per IJJ, while unsynchronized IJJs closer to the substrate (thermal bath) arise higher voltage per IJJ because of the negative temperature dependence of the quasiparticle resistance. The excess voltage due to the unsynchronized IJJs is higher at lower $T_{b}$. Their argument is also valid for the present result, where the bias voltage is higher than the value estimated from the emission frequency via the ac Josephson relation as plotted as open symbols in Fig. 4(a). Since the behavior of $f_{e}-\bar{R}_{c}$ plots looks irrelevant to the deviation from the ac Josephson relation (solid line), we consider that the present results indicate both in-plane and out-of-plane strong temperature inhomogeneities.

\section{SUMMARY}

This study demonstrated the qualitative difference in the temperature dependence of the frequencies emitted by usual mesa IJJ stacks and a stand-alone IJJ stack. These trends were predicted in previous work by Benseman et al. ${ }^{15} \mathrm{By}$ considering a toy model, we attributed the significant bias tuning at a fixed bath temperature to inhomogeneous temperature rise of the stack (relative to the bath temperature). The linewidths of the emission spectra were less than $1 \mathrm{GHz}$, regardless of emission type. These results strongly contrast with those of Li et al., ${ }^{19}$ who reported poor synchronization of stacked IJJs in the low-bias region.

\section{ACKNOWLEDGMENTS}

The authors acknowledge M. Tsujimoto, A. Elarabi, Y. Maeda, Y. Kamei, T. Nakagawa, Y. Nakagawa, S. Mizuta, T. Yamamoto, and K. Kadowaki for their technical 
assistance. Comments from A. Koshelev, T. Koyama, and X. $\mathrm{Hu}$ are greatly appreciated. This work was supported by KAKENHI (23681030).

${ }^{1}$ L. Ozyuzer, A. E. Koshelev, C. Kurter, N. Gopalsami, Q. Li, M. Tachiki, K. Kadowaki, T. Yamamoto, H. Minami, H. Yamaguchi, T. Tachiki, K. E. Gray, W. K. Kwok, and U. Welp, Science 318, 1291 (2007).

${ }^{2}$ K. Kadowaki, H. Yamaguchi, K. Kawamata, T. Yamamoto, H. Minami, I. Kakeya, U. Welp, L. Ozyuzer, A. E. Koshelev et al., Phys. C 468, 634 (2008).

${ }^{3}$ S. Lin and X. Hu, Phys. Rev. Lett. 100, 247006 (2008).

${ }^{4}$ H. Minami, I. Kakeya, H. Yamaguchi, T. Yamamoto, and K. Kadowaki, Appl. Phys. Lett. 95, 232511 (2009).

${ }^{5}$ H. B. Wang, S. Guénon, J. Yuan, A. Iishi, S. Arisawa, T. Hatano, T. Yamashita, D. Koelle, R. Kleiner, and S. Guenon, Phys. Rev. Lett. 102, 017006 (2009), e-print arXiv:0807.2749.

${ }^{6}$ T. Koyama, H. Matsumoto, M. Machida, and K. Kadowaki, Phys. Rev. B 79, 104522 (2009).

${ }^{7}$ X. Hu and S.-Z. Lin, Supercond. Sci. Technol. 23, 053001 (2010).

${ }^{8}$ U. Welp, K. Kadowaki, and R. Kleiner, Nat. Photonics 7, 702 (2013).

${ }^{9}$ K. Yamaki, M. Tsujimoto, T. Yamamoto, A. Furukawa, T. Kashiwagi, H. Minami, and K. Kadowaki, Opt. Express 19, 3193 (2011), e-print arXiv: $1005.2341 \mathrm{v} 1$.

${ }^{10}$ S. Sekimoto, C. Watanabe, H. Minami, T. Yamamoto, T. Kashiwagi, R. A. Klemm, and K. Kadowaki, Appl. Phys. Lett. 103, 182601 (2013).

${ }^{11}$ T. M. Benseman, K. E. Gray, A. E. Koshelev, W.-K. Kwok, U. Welp, H. Minami, K. Kadowaki, and T. Yamamoto, Appl. Phys. Lett. 103, 022602 (2013).

${ }^{12}$ A. Yurgens, Phys. Rev. B 83, 184501 (2011).

${ }^{13}$ I. Kakeya, Y. Omukai, T. Yamamoto, K. Kadowaki, and M. Suzuki, Appl. Phys. Lett. 100, 242603 (2012).

${ }^{14}$ M. Tsujimoto, H. Kambara, Y. Maeda, Y. Yoshioka, Y. Nakagawa, and I. Kakeya, Phys. Rev. Appl. 2, 044016 (2014).
${ }^{15}$ T. M. Benseman, A. E. Koshelev, K. E. Gray, W.-K. Kwok, U. Welp, K. Kadowaki, M. Tachiki, and T. Yamamoto, Phys. Rev. B 84, 064523 (2011).

${ }^{16}$ K. Wiesenfeld, P. Colet, and S. Strogatz, Phys. Rev. Lett. 76, 404 (1996).

${ }^{17}$ H. Wang, S. Guénon, B. Gross, J. Yuan, Z. Jiang, Y. Zhong, M. Grünzweig, A. Iishi, P. Wu, T. Hatano, D. Koelle, and R. Kleiner, Phys. Rev. Lett. 105, 057002 (2010).

${ }^{18}$ M. Tsujimoto, T. Yamamoto, K. Delfanazari, R. Nakayama, T. Kitamura, M. Sawamura, T. Kashiwagi, H. Minami, M. Tachiki, K. Kadowaki, and R. A. Klemm, Phys. Rev. Lett. 108, 107006 (2012).

${ }^{19}$ M. Li, J. Yuan, N. Kinev, J. Li, B. Gross, S. Guénon, A. Ishii, K. Hirata, T. Hatano, D. Koelle, R. Kleiner, V. Koshelets, H. Wang, and P. Wu, Phys. Rev. B 86, 060505 (2012).

${ }^{20}$ I. Kakeya, N. Hirayama, T. Nakagawa, Y. Omukai, and M. Suzuki, Phys. C 491, 11 (2013).

${ }^{21}$ R. Kleiner, Phys. Rev. B 50, 6919 (1994).

${ }^{22}$ W. Anukool, S. Barakat, C. Panagopoulos, and J. R. Cooper, Phys. Rev. B 80, 024516 (2009).

${ }^{23}$ T. Jacobs, S. Sridhar, Q. Li, G. Gu, and N. Koshizuka, Phys. Rev. Lett. 75, 4516 (1995).

${ }^{24}$ S.-F. Lee, D. Morgan, R. Ormeno, D. Broun, R. Doyle, J. Waldram, and K. Kadowaki, Phys. Rev. Lett. 77, 735 (1996).

${ }^{25}$ T. Kashiwagi, K. Yamaki, M. Tsujimoto, K. Deguchi, N. Orita, T. Koike, R. Nakayama, H. Minami, T. Yamamoto, R. A. Klemm, M. Tachiki, and K. Kadowaki, J. Phys. Soc. Jpn. 80, 094709 (2011).

${ }^{26}$ B. Gross, J. Yuan, D. Y. An, M. Y. Li, N. Kinev, X. J. Zhou, M. Ji, Y. Huang, T. Hatano, R. G. Mints, V. P. Koshelets, P. H. Wu, H. B. Wang, D. Koelle, and R. Kleiner, Phys. Rev. B 88, 014524 (2013).

${ }^{27}$ H. Minami, C. Watanabe, K. Sato, S. Sekimoto, T. Yamamoto, T. Kashiwagi, R. A. Klemm, and K. Kadowaki, Phys. Rev. B 89, 054503 (2014).

${ }^{28}$ T. M. Benseman, A. E. Koshelev, W.-K. Kwok, U. Welp, K. Kadowaki, J. R. Cooper, and G. Balakrishnan, Supercond. Sci. Technol. 26, 085016 (2013). 